\title{
DETERMINACIÓN DEL CONTENIDO DE CADMIO EN SUELOS, FRUTOS, GRANOS FERMENTADOS Y SECOS, LICOR DE CACAO Y CHOCOLATE EN ZONAS PRODUCTORAS DE LA REGIÓN SAN MARTÍN
}

\author{
Wilson Santander Ruiz ${ }^{* a}$, Richer Garay Montes ${ }^{\mathrm{a}}$, Carlos Verde Girbau $^{\mathrm{b}}$, \\ Oscar Mendieta Taboada ${ }^{\mathrm{a}}$
}

\begin{abstract}
RESUMEN
El contenido de cadmio en suelos, hojas, almendras frescas (testa y embrión), almendras fermentadas secas, licor de cacao y tabletas de chocolate con $70 \%$ de cacao, de dos provincias (Bellavista y Huallaga) y cinco localidades (3 en Bellavista y 2 en Huallaga) de la Región San Martín, fue evaluado en el presente trabajo. El contenido de cadmio se determinó por espectrofotometría de absorción atómica, utilizando muestra filtrada obtenida de $0,5 \mathrm{~g}$ de muestra seca y $5 \mathrm{ml}$ de solución nítrica perclórica, previa digestión; ensayos realizados en laboratorios de la Universidad Nacional de San Martín (UNSM). Los valores máximos obtenidos para contenido de cadmio (ppm) fueron: en suelos, 0,960; en hojas 0,780; en testa y cotiledón 0,098; en almendras fermentadas y secas 0,075; en licor de cacao, 0,210; en tabletas de chocolate al $70 \%$ de cacao 0,625. El coeficiente de correlación $\left(\mathrm{R}^{2}\right)$ para almendras fermentadas y secas indica que no existe diferencia significativa entre zonas, mientras que en licor de cacao la muestra de Tingo de Saposoa fue estadísticamente diferente $(\mathrm{p}<0,05)$, estando todos los valores por debajo del máximo permisible. El incremento del contenido de cadmio en chocolate puede deberse a los ingredientes complementarios (leche, panela) o al equipo de procesamiento utilizado.
\end{abstract}

Palabras clave: Theobroma cacao, cadmio, metales pesados.

\footnotetext{
a Facultad de Ingeniería Agroindustrial, Escuela Profesional de Ingeniería Agroindustrial, Universidad Nacional de San Martín - Tarapoto.*Autor de correspondencia: esantander@unmsm.edu.pe; celular: 945499416

b Facultad de Ciencias Agrarias, Escuela Profesional de Agronomía, Universidad Nacional de San Martín Tarapoto.
} 


\title{
DETERMINATION OF THE CADMIUM CONTENT IN SOILS, FRUITS, FERMENTED AND DRY BEANS, COCOA LIQUOR, AND CHOCOLATE IN PRODUCING AREAS OF THE SAN MARTÍN REGION
}

\begin{abstract}
In the present work was evaluated the content of cadmium in soils, leaves, fresh almonds (testa and embryo), dried fermented almonds, cocoa liquor, and chocolate bars with $70 \%$ cocoa, two provinces (Bellavista and Huallaga), and 5 locations ( 3 in Bellavista and 2 in Huallaga) of the San Martín Region. The cadmium content was determined with an atomic absorption spectrophotometer, using a filtered sample obtained from $0.5 \mathrm{~g}$ of dry sample and $5 \mathrm{ml}$ of perchloric nitric solution, after digestion; tests carried out in laboratories of the Universidad Nacional de San Martín (UNSM). The maximum values obtained for cadmium content (ppm) were: in soils, 0.960 ; in sheets 0.780 ; in testa and cotyledon 0.098 ; in fermented and dried almonds 0.075 ; in cocoa liquor, 0.210 ; in chocolate tablets with $70 \%$ cocoa 0.625 . The correlation coefficient $\left(\mathrm{R}^{2}\right)$ for fermented and dried almonds, indicates that there is no significant difference between zones, while in cocoa liquor the Tingo de Saposoa sample was statistically different $(\mathrm{p}<0.05)$, all values being below the maximum allowable. The increase in the content of cadmium in chocolate may be due to complementary ingredients (milk, panela) or to the processing equipment used.
\end{abstract}

Key words: Theobroma cacao, cadmium, heavy metals.

\section{INTRODUCCIÓN}

Una de las principales actividades de importancia socio-económica de la región San Martín y otras regiones del país es el cultivo del cacao (Theobroma cacao L.). Entre los mercados más importantes para este producto, y sus derivados, se puede mencionar a los Estados Unidos y Europa (Francia, Inglaterra, Bélgica). Un riesgo potencial para la salud de los seres humanos lo constituye la ingesta de cadmio, metal que al estar presente en la corteza terrestre puede ser absorbido por las plantas y, a través de ellas, llegar al ser humano.

Los contenidos de cadmio que superan los valores permitidos $(0,5 \mathrm{ppm})$, limitan la exportación de cacao. El cadmio es un elemento de relativa abundancia en la naturaleza y puede liberarse al ambiente de diferentes formas, entre ellas actividades naturales como la actividad volcánica. La presencia de cadmio en chocolate y productos derivados del cacao es un tema de gran importancia pues causa un fuerte impacto en la economía de los países productores ${ }^{1}$. En la 77ma. Reunión del Comité Mixto de Expertos en Aditivos Alimentarios ${ }^{2}$ de la FAO/ OMS, realizada en Roma, este organismo ha resaltado que la exposición al cadmio en dietas con elevados niveles de consumo de cacao y productos del cacao ha sido aparentemente sobrestimada, sin embargo el hecho de no haberse establecido un Nivel Máximo (NM) de cadmio para chocolate y productos de cacao podría constituirse en una amenaza para las 
exportaciones de algunos países, en particular para aquellos que son exportadores de cacao, como es el caso del Perú.

La Autoridad Europea de Seguridad Alimentaria ${ }^{3}$, a través de su Comité sobre Contaminantes de los Alimentos, ha dictaminado que la exposición alimentaria al cadmio en países de Europa está próxima o supera ligeramente el nivel de ingesta semanal tolerable de $2,5 \mu \mathrm{g} / \mathrm{kg}$ de peso corporal. De forma concordante, la Comisión del Codex Alimentarius de FAO/OMS4 acordó proponer los niveles máximos de cadmio para chocolates y derivados siguientes: $0,20 \mathrm{ppm}$, para chocolate con leche y contenido de materia seca total de cacao menor al 30\%; 0,60 ppm para chocolate con contenido de materia seca total de cacao inferior al $50 \%$ y para chocolate con leche con un contenido de materia seca total de cacao igual o mayor al $30 \%$; $1,5 \mathrm{ppm}$ para cacao en polvo vendido al consumidor final o para ser empleado como ingrediente en la elaboración de cacao en polvo edulcorado que es vendido al consumidor final (chocolate para beber); 2,0 ppm para chocolate con contenido de materia seca total de cacao igual o superior a $50 \%$.

La Unión Europea, al anunciar la aplicación de regulaciones al contenido de cadmio (Cd) en chocolate y productos de cacao, ha generado preocupación entre los agricultores dedicados a la producción de cacao en todo el mundo. La Unión Europea ha recomendado un contenido máximo de cadmio de $0,8 \mathrm{mg} / \mathrm{kg}^{4}$, para chocolate con un contenido igual o mayor al $50 \%$ de sólidos de cacao.

Los países de América Latina están considerados entre los principales productores de cacao, existiendo alrededor de 500000 productores de cacao en la región. A nivel mundial, se tiene cerca de tres millones y medio de pequeños agricultores cuya economía familiar está basada en la producción de cacao. El cacao fino alcanza en la actualidad del $6 \%$ al $8 \%$ de la producción mundial de cacao, con un gran aporte de los países de América Latina y el Caribe, que representa cerca del $80 \%$ de la producción mundial de dicho tipo de cacao. Ecuador, con el $54 \%$ de la producción mundial, es el mayor productor de cacao fino, ubicándose en posiciones importantes República Dominicana y Perús.

En cuanto a las importaciones netas de cacao, las de países europeos representan el $58 \%$, de Norte América, $27 \%$, de Asia $14 \%$ y de África $2 \%$. El mayor importador mundial es Estados Unidos, con un volumen que representa el $20 \%$ de las importaciones globales netas, ubicándose después Alemania (13\%), Bélgica (7\%), Francia (6 \%) y la Federación Rusa (6 \%). La gran mayoría de importaciones de granos de cacao que realiza Europa proviene de África Occidental, con un $93 \%$, mientras que las importaciones provenientes de América Latina y del sureste asiático son consideradas de nivel secundario y terciario, respectivamente ${ }^{1}$.

Considerando que el contenido elevado de metales pesados, entre ellos cadmio, en el cacao es limitante en su demanda en el mercado nacional e internacional, el presente trabajo tuvo como objetivo determinar el contenido de cadmio en suelos, hojas, frutos (frescos y secos), licor de cacao y chocolate en zonas productoras de las provincias de Bellavista y Huallaga de la región San Martín. 


\section{PARTE EXPERIMENTAL}

Las muestras de suelos, hojas, frutos (almendras frescas y secas) fueron obtenidas de las fincas de 25 agricultores cacaoteros ( 5 por localidad) en el año 2016, pertenecientes a las zonas de Huingoyacu, Panamá y Tingo de Saposoa en la provincia del Bellavista y Ahuihua y Tánger en la provincia del Huallaga (figura 1), para lo cual se contó con el apoyo del personal de campo de la Cooperativa Agraria Cacaotera ACOPAGRO Ltda.

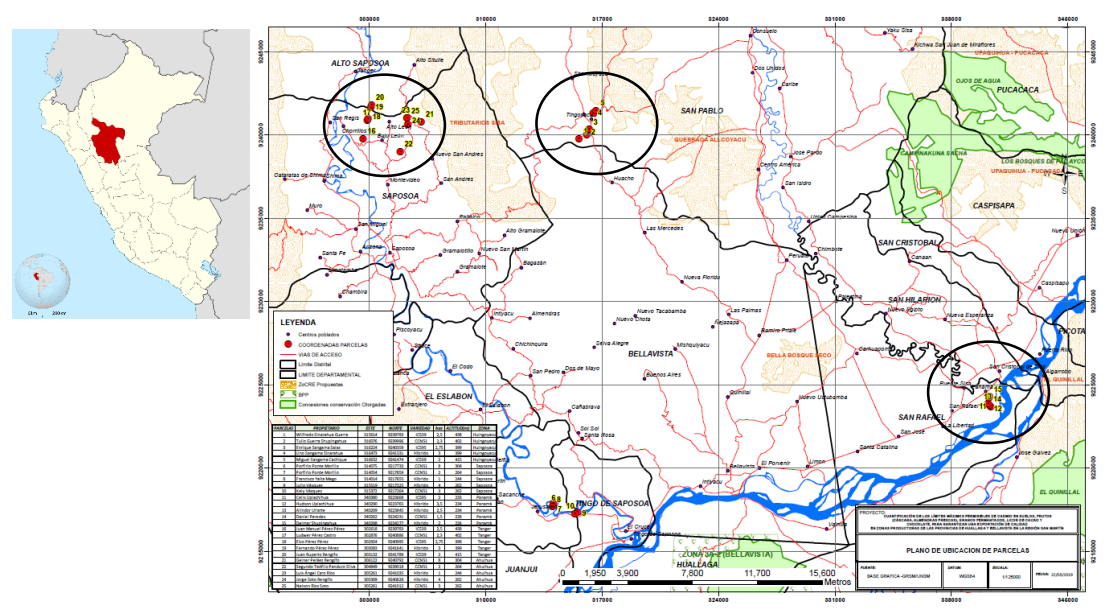

Figura 1. Ubicación de las localidades intervenidas en el estudio.

Asimismo, estas muestras recolectadas fueron destinadas al Laboratorio de Análisis de Suelos, Aguas y Foliares de la Facultad de Ciencias Agrarias (FCA) de la UNSM para los análisis físico-químicos respectivos, y una parte de las almendras secas se derivó al Laboratorio de Control de Calidad de la Cooperativa ACOPAGRO para el acondicionamiento y elaboración del licor de cacao y de las tabletas de chocolate al $70 \%$ de contenido de cacao; una parte de las muestras de licor de cacao y tabletas de chocolate se enviaron al Laboratorio de Análisis de Suelos, Aguas y Foliares de la FCA para el análisis físico-químico respectivo ( $\mathrm{pH}$, conductividad eléctrica y cadmio).

Los equipos y materiales que se emplearon fueron: balanza analítica, marca BOECO, modelo BBX 31; potenciómetro marca SI Analytics, modelo LAB 850; hidrómetro de Bouyoucos, marca THERMCO, modelo GW152H; conductímetro, marca SI Analytics, modelo LAB 960; estufa, marca ECOCCEL, modelo 111 ECO; espectrofotómetro de absorción atómica, marca GBC, modelo SavantAA; digestor microkjeldhal, marca JP SELECTA, modelo RAT; destilador de nitrógeno, marca RAT, modelo Pro Nitro M; espectrofotómetro UV visible, marca UNICO, modelo 1205 VIS. 
El método AOAC 974.276 fue empleado para determinar el contenido de cadmio. Se utilizó un espectrofotómetro de absorción atómica marca GBC SavantAA, aplicando una muestra filtrada a partir de $0,5 \mathrm{~g}$ de muestra seca por $5 \mathrm{ml}$ de solución nítrica perclórica, previa digestión en equipo Digestor Trade Raypa de 12 posiciones, análisis realizado en el Laboratorio de Suelos, Aguas y Foliares perteneciente a la Facultad de Ciencias Agrarias (FCA) de la Universidad Nacional de San Martín (UNSM).

Los resultados obtenidos fueron procesados con el paquete estadístico SAS V9.2 (2012), efectuándose los análisis de correlación de Pearson entre los contenidos de cadmio presentes en el suelo, fruto (almendras frescas con testa y sin testa), granos fermentados, licor de cacao y chocolate.

\section{RESULTADOS Y DISCUSIÓN}

En los suelos de las cinco localidades consideradas (figura 2), pertenecientes a las provincias de Bellavista y Huallaga, el contenido de cadmio presentó niveles mínimos de 0,214 ppm y máximos de 0,980 ppm, que son mucho menores que los determinados en parcelas ubicadas en la región Huánuco ${ }^{7}$, cuyos suelos presentaron valores más elevados de cadmio disponible (1,82 y 1,63 ppm) en sectores cercanos a las riberas de los ríos Tulumayo y Huallaga, respectivamente. En la región San Martín, la zona de Panamá reporta valores de cadmio por debajo de los límites máximos permisibles, sin embargo, otras zonas como Huingoyacu, Tingo de Saposoa, Tanger y Ahuihua, presentan valores por encima de los indicados por García y Dorronsoro ${ }^{8}$.

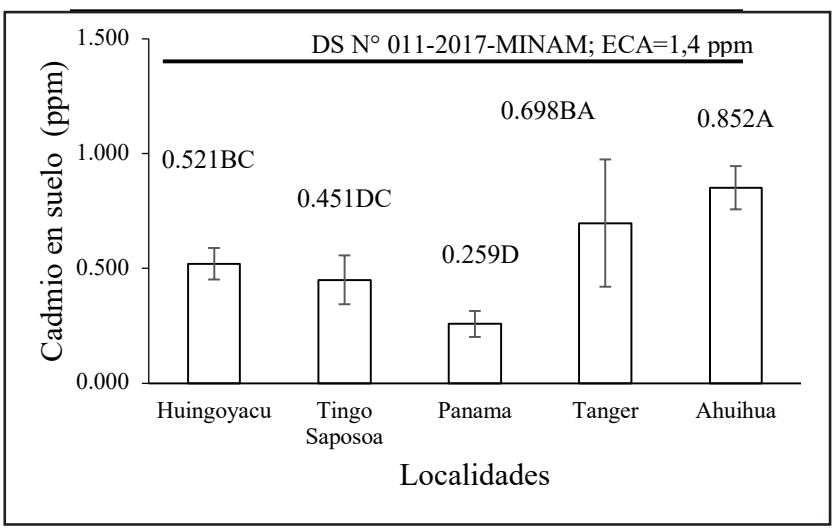

Figura 2. Contenido de cadmio (ppm) en suelo en diferentes localidades. 


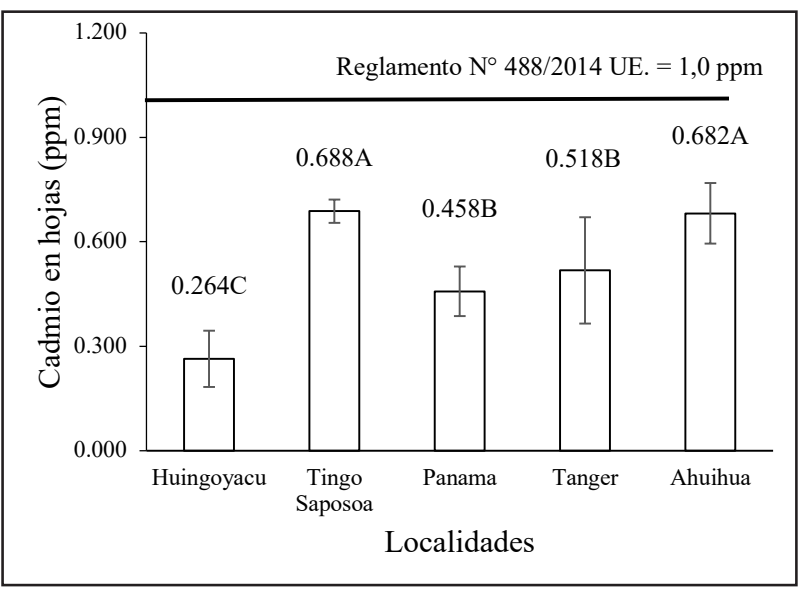

Figura 3. Contenido de cadmio (ppm) en hojas en diferentes localidades.

Respecto al contenido de cadmio en hojas o tejido foliar (figura 3) se encontraron niveles mínimos de 0,18 ppm y máximos de 0,780 ppm, obteniéndose un promedio estimado de 0,49 ppm, que es ligeramente inferior a la concentración máxima tolerable de cadmio determinada en hojas maduras ${ }^{9}$, del orden de $0,5 \mathrm{ppm}$. De otro lado, el valor promedio obtenido de 0,49 ppm es inferior al encontrado en material foliar ${ }^{7}$ que alcanzó 0,66 ppm y al determinado en hojas maduras de ocho diferentes clones de cacao (ICS-6, ICS-1, IMC-67, ICS-95, SCA-6, UF-29, POUND-12 y CCN-51) comprendidos en el rango de 0,54 ppm a 0,89 $\mathrm{ppm}^{10}$. Asimismo, los valores máximo, promedio y mínimo para contenido de cadmio en hojas de cacao, obtenidos en el presente trabajo, son cercanos a los reportados para muestras recolectadas en territorio de la comunidad nativa Pakun, anexo Chiriaco, distrito Imaza, provincia de Bagua $^{11}$, de 0,087, 0,509 y <0,008 mg.kg-1, para máximo, promedio y mínimo, respectivamente.

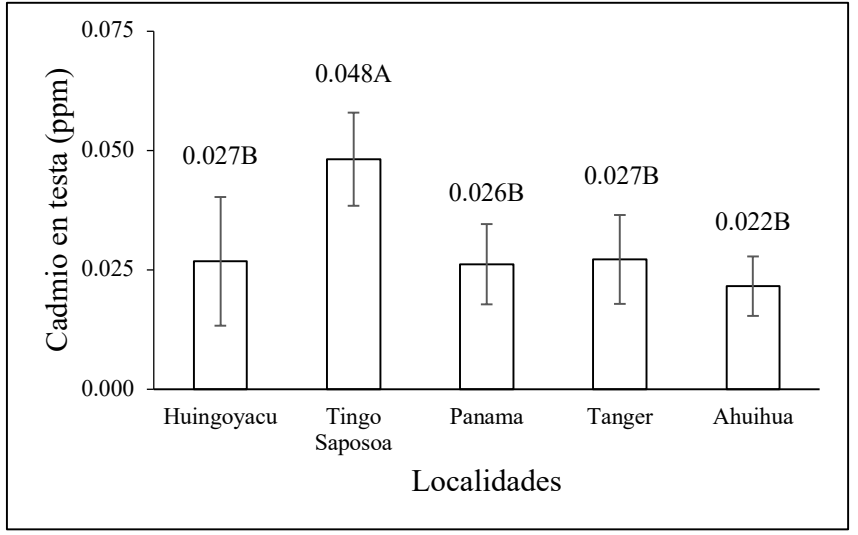

Figura 4. Contenido de cadmio (ppm) en Testa en diferentes localidades. 


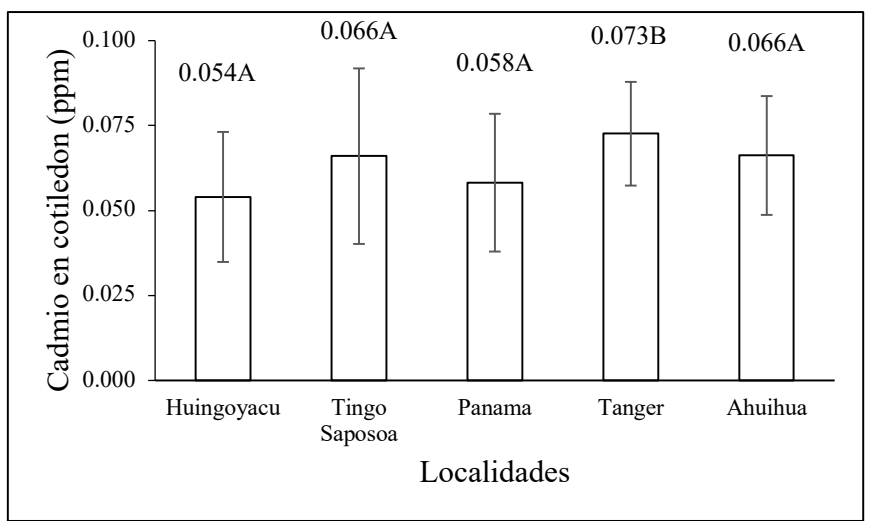

Figura 5. Contenido de cadmio (ppm) en cotiledón en diferentes localidades.

El contenido de cadmio en testa (figura 4) presentó niveles mínimo y máximo de 0,012 y 0,056, respectivamente. Estos valores son mucho menores a los reportados para cacao de Costa de Marfil ${ }^{12}$ que están en el rango de 0,343 ppm a 0,644 ppm. En cotiledón (figura 5), el valor mínimo de contenido de cadmio fue de 0,021 ppm y el máximo de 0,098 ppm; estos valores son cercanos a los determinados en cacao de Costa de Marfil ${ }^{12}$, en el rango de 0,018 ppm a 0,088 ppm de cadmio, e inferiores a los reportados para ocho clones de cacao del Jardín interclonal de la Universidad Nacional de Ucayali ${ }^{10}$, de 0,33 ppm, para CCN-51, a 1,18 ppm para ICS-1.

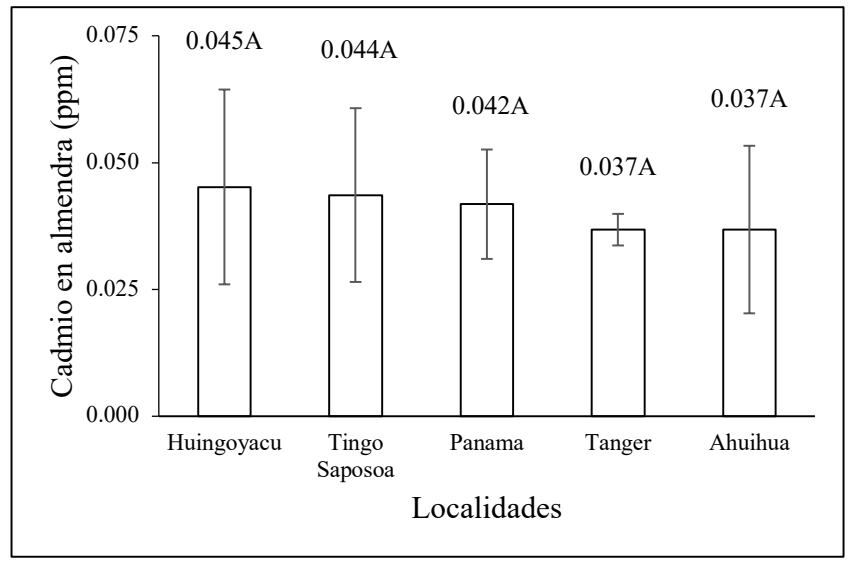

Figura 6. Contenido de cadmio (ppm) en almendras de diferentes localidades. 
El contenido de cadmio encontrado en las almendras fermentadas y secas (figura 6) presentó valores mínimos de 0,023 ppm y máximos de 0,075 ppm, no encontrándose diferencia significativa entre las zonas consideradas $(\mathrm{p}>0,05)$. Los valores obtenidos son inferiores a los determinados en estudios realizados en la región San Martín ${ }^{13}$, reportándose valores de cadmio entre $0,1 \mathrm{ppm}$ y 2,45 ppm. Asimismo, los valores encontrados en el presente trabajo son inferiores al determinado en almendras de frutos de cacao provenientes de parcelas bajo cultivo orgánico ${ }^{7}$, que alcanzó $1,55 \mathrm{ppm}$, y al informado para almendras de cacao cultivadas en el sur de Ecuador ${ }^{14}$, en las cuales se determinaron valores en el rango de 0,02 a 3,00 ppm, con promedio de 0,94 ppm, considerados por encima del nivel crítico. Se debe tener en cuenta que el nivel crítico de referencia para almendras de cacao es de $0,5 \mathrm{ppm}^{8}$.

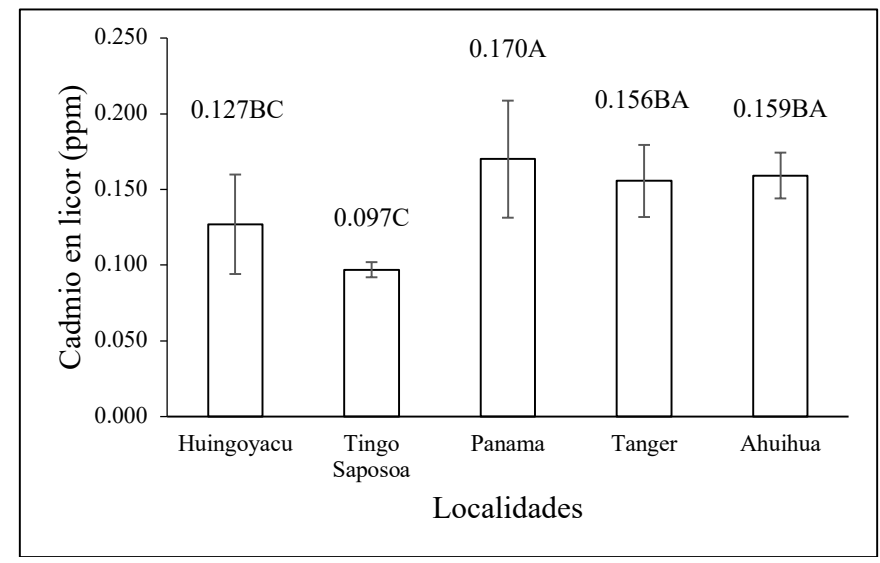

Figura 7. Contenido de cadmio (ppm) en licor de cacao en diferentes localidades.

Con respecto al licor de cacao obtenido a partir de los granos fermentados y secos de cacao de las 5 localidades y 2 provincias de la región San Martín (figura 7), se encontró niveles mínimos de cadmio de 0,089 ppm y máximos de 0,210 ppm, siendo la muestra obtenida con cacao de Tingo de Saposoa estadísticamente diferente $(\mathrm{p}<0,05)$. Todos los valores obtenidos están muy por debajo del valor promedio de $0,865 \mathrm{ppm}$ reportado para muestras elaboradas con cacao de Malasia ${ }^{15}$. 


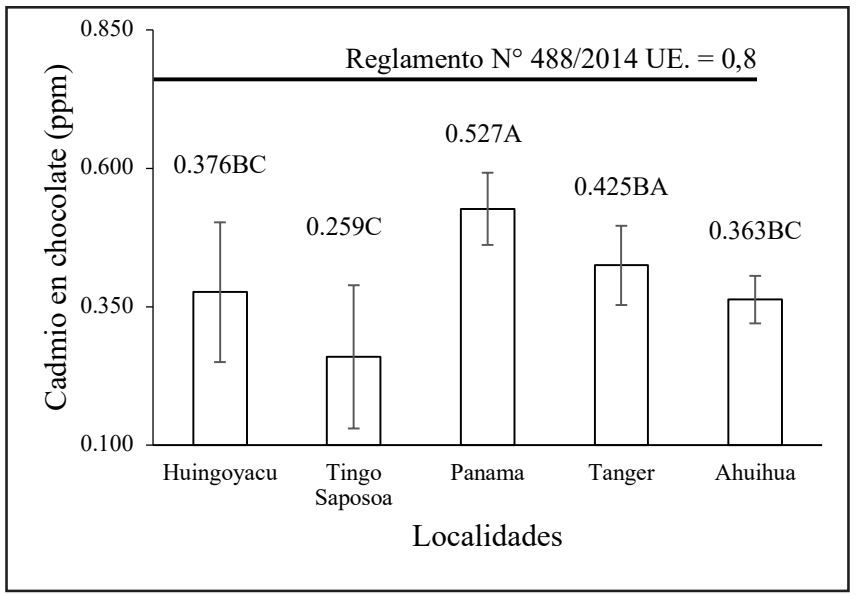

Figura 8. Contenido de cadmio (ppm) en chocolate con cacao de las diferentes localidades.

Para tabletas de chocolate con $70 \%$ de cacao (figura 8), se encontraron niveles mínimos de 0,116 ppm y máximos de 0,625 ppm y las tabletas de chocolate con mayor contenido de cadmio procedieron de muestras de las zonas de Tánger, concordando con valores elevados de cadmio en suelo y hojas, determinados previamente. El valor máximo obtenido $(0,625 \mathrm{ppm})$, es inferior al nivel máximo de $1 \mathrm{mg} / \mathrm{kg}$ de cadmio establecido en Malasia para chocolate ${ }^{15}$.

\section{CONCLUSIONES}

La presencia de metales pesados (cadmio), se evaluó en suelos, hojas, almendras frescas (testa y embrión), almendras fermentadas secas, licor de cacao y tabletas de chocolate con $70 \%$ de contenido de cacao, en dos provincias (Bellavista y Huallaga) y cinco localidades (3 en la provincia de Bellavista y 2 en la provincia de Huallaga); encontrándose la presencia de cadmio en todas las parcelas de las cinco localidades muestreadas en las siguientes cantidades promedios: 0,556 ppm en suelos; 0,522 ppm en hojas; 0,063 ppm en cotiledón; 0,030 ppm en testa; 0,041 ppm en granos fermentados secos; 0,142 ppm en licor de cacao y 0,390 ppm en tabletas de chocolate con leche con $70 \%$ de licor de cacao. Por lo que se concluye que estos niveles obtenidos en granos, licor de cacao y tabletas están dentro de los promedios aceptables y no superan el nivel máximo de cadmio para chocolates y derivados, acordado por el Codex Alimentarius para la Unión Europea.

Las zonas con mayor contenido promedio de cadmio en relación a suelos, diferentes partes de la planta, licor y tabletas de chocolate son las siguientes: Ahuihua $(0,312 \mathrm{ppm})$, Tánger $(0,276$ ppm) en la provincia de Huallaga; seguido de Tingo de Saposoa (0,236 ppm), Panamá (0,220 ppm) y Huingoyacu (0,202 ppm), todos estos pertenecientes a la provincia de Bellavista. 


\section{AGRADECIMIENTO}

A la Universidad Nacional de San Martín-T, por el apoyo brindado al proyecto de investigación. A la Cooperativa Agraria Cacaotera ACOPAGRO Ltda., ubicada en la ciudad de Juanjuí, en su Gerente General Econ. Gonzalo Ríos Núñez; Jefe de Control de Calidad Ing. David Contreras Monjarás; Bach. Evelyn Guevara Santillán; Ing. Katia Vanesa Panduro Soto; Bach. Genovith Venturo Minauro y egresado Jean Stewar Hidalgo Soto de la carrera profesional de Ingeniería Agroindustrial quienes prestan sus servicios a la cooperativa y en esta oportunidad con su apoyo desinteresado en cuanto a la caracterización de los granos y licor de cacao en el laboratorio de Control de Calidad de la mencionada Cooperativa. A los responsables de campo de esta prestigiosa cooperativa; Ing. Miguel Trujillo Valderrama e Ing. Hernán García Meza, quienes apoyaron desinteresadamente en la obtención de muestras de campo y centros de acopio de granos de cacao de las cinco localidades en estudio. Finalmente, a la Ing. Marilyn Paola Zegarra García por la elaboración de los mapas de georreferenciación.

\section{REFERENCIAS BIBLIOGRÁFICAS}

1. International Cocoa Organization (ICCO). The world cocoa economy: past and present. One hundred and forty-sixth meeting. London, 18-21 September 2012, EX/146/7; 2012.

2. Joint FAO/WHO Expert Committee on Food Additives (JECFA). Seventy-seventh meeting. Summary and Conclusions. Rome, 4-13 June; 2013.

3. European Food Safety Authority (EFSA). Cadmium dietary exposure in the European population. EFSA Journal 2012; 10(1): 2551. doi:10.2903/j.efsa.2012.2551.

4. FAO/OMS. Programa Conjunto FAO/OMS sobre Normas Alimentarias. Comité del Códex sobre Contaminantes de los Alimentos. Anteproyecto de Niveles Máximos para el Cadmio en el chocolate y productos derivados de cacao, 9na. Reunión. Nueva Delhi; 16-20 marzo 2015.

5. Escuela Superior Politécnica del Litoral (ESPOL). Estudios industriales. Orientación estratégica para la toma de decisiones. Industria de Cacao. Guayaquil: Escuela Superior Politécnica del Litoral; 2016.

6. AOAC International. Official Method 974.27-Natural mineral waters. En: AOAC International, Official Methods of Analysis. Gaithersburg: AOAC International; 1998.

7. Cárdenas A. Presencia de cadmio en algunas parcelas de cacao orgánico de la cooperativa Agraria Industrial Naranjillo, Tingo María, Perú. [Tesis de pregrado]. Tingo María: Universidad Nacional Agraria de la Selva; 2012.

8. García I, Dorronsoro C. Contaminación por metales pesados. [Internet] Tecnología de Suelos. Universidad de Granada. Departamento de Edafología y Química Agrícola; 2005. [Citado 12 nov 2020]. Disponible en: http://edafologia.ugr.es/conta/tema15/introd.htm

9. Kabata-Pendias A, Pendias H. Trace elements in soils and plants. Boca Raton, Florida: CRC Press; 2001. 
10. López A, Cartagena T, Vásquez R. Niveles de concentración de cadmio $(\mathrm{Cd})$ y plomo $(\mathrm{Pb})$ en el suelo, hojas y almendras de ocho clones de cacao (Theobroma cacao L.) en el Jardín Interclonal de la Universidad Nacional de Ucayali. Pucallpa: Universidad Nacional de Ucayali; 2018.

11. Oc W, Gonza C, Guzmán W, Pariente E. Bioacumulación de cadmio en el cacao (Theobroma cacao) en la Comunidad Nativa de Pakun, Perú. Revista Forestal del Perú 2018; 33(1): 63-75.

12. Yapo KD, Ouffoue SK, N'Guessan BR, Okpekon TA, Dade J, Say M, et al. Quality control by the determination of heavy metals in new variety of cocoa (cocoa mercedes) in Côte d'Ivoire. J Soc Ouest-Afr Chim. 2014; 037: 56-64.

13. Instituto de Cultivos Tropicales/Programa de Desarrollo Rural Sostenible de la Cooperación Técnica Alemana (ICT/PDRS-GTZ). Monitoreo de la presencia de cadmio en almendras de cacao, suelos y agua en San Martín y Amazonas. Tarapoto; 2008.

14. Chávez E, He ZL, Stoffella PJ, Mylavarapu RS, Li YC, Moyano B, et al. Concentration of cadmium in cacao beans and its relationship with soil cadmium in southern Ecuador. Sci Total Environ. 2015; 533: 205-214.

15. Lee CK, Low KS. Determination of Cadmium, Lead, Copper and Arsenic in Cocoa, Semi-Finished and Finished Chocolate Products. Pertanika 1985; 8: 243-248. 